\title{
ON FURTHER PROPERTIES OF IMPROJECTIVE OPERATORS
}

\author{
E. TARAFDAR \\ (Received 9 February 1971) \\ Communicated by J. B. Miller
}

\section{Introduction}

This is a continuation of our work [4]. The purpose of this paper is to find the relation of the class of improjective operators on some Banach space, with the classes of strictly singular, strictly cosingular and $\phi$-admissible perturbations on $X$ and to investigate those pairs of Banach spaces for which all bounded linear operators having either of the pair as domain and the other as range are improjective, or strictly singular. The beginning of Section 1 is intended to familiarize the reader with the concepts and notations used in this paper.

In Section 2 we have proved that on any Banach space an inessential operator introduced by Kleinecke [8] is an improjective operator. We have also deduced that the class of inessential operators and the class of improjective operators coincide with the class of strictly singular operators on a subprojective Banach space and coincide with the class of strictly consingular operators on a reflexive and superprojective Banach space. We have also obtained that if $X$ is a Banach space such that the class $P(X)$ of improjective operators on $X$ is included in the class $R(X)$ of Riesz operators on $X$, then $P(X)$ coincides with the class $I(X)$ of inessential operators. We have given an example of a Banach space $X$ where $R(X)$ is not included in $P(X)$. It is known [5] that if $X=c_{0}$, or $l^{p}(1 \leqq p<\infty)$, then the compact operators form the largest ideal in the ring of all bounded linear operators on $X$. In this section we have proved that if $X=m$ then the improjective operators form the largest ideal in the semigroup of all bounded linear operators on $X$.

In the beginning of Section 3 is listed a class of pairs of Banach spaces such that for each such pair all bounded linear operators having either of the pair as the domain and the other as the range are improjective. We have also deduced some corollaries, including

(i) If $X$ is a Banach space isomorphic to an abstract $L$-space and $Y=c_{0}$, or $l^{p}(S)(1<p<\infty)$, or $L^{p}(2 \leqq p<\infty)$, or $C\left(S^{\prime}\right)\left(\right.$ where $S^{\prime}$ is metrizable, 
compact and dispersed), and $Y^{\prime}=l^{p}(S)(1<p<\infty)$, or $L_{p}(1<p \leqq 2)$, then every bounded linear operator of $X$ into $Y$ is strictly singular and every bounded linear operator of $Y^{\prime}$ into $X$ is strictly cosingular

(ii) If $X \in P$ (for definition see Section 3) and $Z=Y$ as in (i) or l(S), $S$ being a countable set and $Y^{\prime}$ has the same meaning as in (i), then every bounded linear operator of $X$ into $Z$ is strictly singular and every bounded linear operator of $Y^{\prime}$ into $X$ is strictly cosigular;

if $X \in P_{1}$ and $U=Z$ as above, or any reflexive Banach space, then every bounded linear operator of $X$ into $U$ is strictly singular.

(iii) Every bounded linear operator of $L_{p}$ into $l^{q}$ where $1<p<\infty, 1 \leqq q$ $<\infty, q \neq 2$ and $p \neq q$ is strictly singular.

This work was carried out under the supervision of Professor B. Abrahamson. The author wishes to thank him for his constant encouragement and advice.

\section{Definitions and notations}

The set of all bounded linear operators from a Banach space $X$ into a Banach space $Y$ will be denoted by $B(X, Y)$.

A subspace $U$ of a Banach space $X$ is said to be complemented in $X$ if it is closed, and there is a closed subspace $V$ of $X$ such that for each $x \in X$ there exist a $u$ in $U$ and $v$ in $V$ such that $x=u+v$ and if $u+v=0$, then $u=v=0$.

We know that a closed subspace $U$ of a Banach space $X$ is complemented in $X$ if and only if there is a projection (a linear idempotent operator) of $X$ onto $U$.

An operator $T \in B(X, Y)$ is called a strictly singular operator if, for no infinite dimensional subspace $M$ of $X$, is the restriction of $T$ to $M$ an isomorphism (see [1] or [2]). The following definition is due to Pelczynski [3];

An operator $T \in B(X, Y)$ is said to be strictly cosingular if, for no infinite dimensional Banach space $Z$ do there exist epimorphisms (onto maps) $T_{1}$ : $X \rightarrow Z$ and $T_{2}: Y \rightarrow Z$ such that the diagram

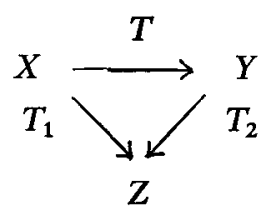

commutes. An operator $T \in B(X, Y)$ is said to be improjective if, for any infinite dimensional closed subspace $M$ of $X$ such that $T M$ is complemented in $Y$, the restriction of $T$ to $M$ is not an isomorphism. 
The following relations hold [4].

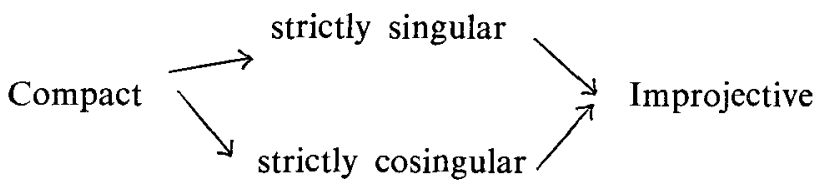

Other properties of improjective operators can be found in [4].

$\phi$-admissible pertubations.

An operator $T \in B(X, Y)$ is called a $\phi$-operator or a Fredholm operator if the range of $T$ is closed in $Y, \alpha(T)=$ the dimension of the null space of $T$ is finite and $\beta(T)=$ the codimension of the range $R(T)$ is finite.

An operator $T \in B(X, Y)$ is called a $\phi$-admissible perturbation or a Fredholm perturbation if $A+T$ is a $\phi$-operator for all $\phi$-operator $A \in B(X, Y)$ (see [5]).

We use the following notations for subsets of $B(X, Y)$;

$P(X, Y)=$ the set of all improjective operators,

$S(X, Y)=$ the set of all strictly singular operators,

$S C(X, Y)=$ the set of all strictly cosingular operators,

$F(X, Y)=$ the set of all $\phi$-admissible perturbations,

$W(X, Y)=$ the set of all weakly compact operators,

$T(X, Y)=$ the set of all compact operators.

$B(X), P(X), S(X)$ etc, will stand repectively for $B(X, X), P(X, X), S(X, X)$ etc.

A Banach space $X$ is said to be subprojective if every infinite dimensional closed subspace $M$ of $X$ contains an infinite dimensional subspace $N$ complemented in $X$. A Banach space $X$ is superprojective if, for every closed subspace $M$ with infinite codimension, there exists a closed subspace $N$ of infinite codimension such that $N$ contains $M$ and is complemented in $X$.

For all other notations used in this paper we refer to [6].

\section{2.}

Let $\operatorname{Rad} A$ denote the Jacobson radical of any ring $A$ and for any Banach space $X$ let

$$
I(X)=\pi^{-1}[\operatorname{Rad}\{B(X) / T(X)\}]
$$

where $\pi$ is the canonical homomorphism of $B(X)$ onto $B(X) / T(X) . I(X)$ is a closed two-sided ideal of $B(X)$, called the ideal of inessential operators (see [7] and $[8]$.

For any Banach space $X$ we have the following characterization of $I(X)$ ([7] p. 604).

For any $T \in B(X), T \in I(X)$ if and only if $I-W T \in \phi_{+}(X)$ and the range of $I-W T$ is complemented in $X$ for all $W \in B(X)$ where $I$ is the identity operator on $X$, 
where $\phi_{+}(X)$ is the class of operators $T$ in $B(X)$ such that $\alpha(T)<\infty$ and $R(T)$ is closed.

From the results of [8] and [9] it easily follows that for any Banach space $X$,

$$
F(X)=I(X) .
$$

(See the proof of lemma 2.1 in [7]).

THEOREM 2.1 For any Banach space $X, I(X) \subseteq P(X)$.

Proof. The proof is similar to that of the theorem 2.2 in [7]. Let $T \in I(X)$ and if possible, let $T \notin P(X)$. Then there is an infinite dimensional closed subspace $X_{1}$ of $X$ such that the restriction $T^{\prime}$ of $T$ to $X_{1}$ is an isomorphism and $T X_{1}$ is complemented in $X$. Since $T X_{1}$ is complemented in $X$, we have $X=T X_{1}+X_{2}$ for some closed subspace $X_{2}$ of $X$. For each $x \varepsilon X$, we define $\bar{T}(x)=T^{\prime-1}\left(x_{1}\right)$ where $x=x_{1}+x_{2}, x_{1} \in T X$, and $x_{2} \in X_{2}$. Clearly $\bar{T} \in B(X)$ and has the property that $\bar{T} \equiv T^{\prime-1}$ on $T X_{1}$ and $\bar{T} \equiv 0$ on $X_{2}$. Now since $T \in I(X), \pi(T) \in \operatorname{Rad} . B(X) / T(X)$. Then by the characterization of $\operatorname{Rad} B(X) / T(X)$ mentioned earlier, $I-\bar{T} T \in \phi_{+}(X)$. Thus the null space of $I-\bar{T} T$ is finite dimensional. Now $\bar{T} T X_{1}=T^{-1} T X_{1}=X_{1}$ is an infinite dimensional closed subspace of $X$ and $(I-\bar{T} T) X_{1}=0$. But this implies that $X_{1}$ is contained in the null space of $I-\bar{T} T$. Thus the null space of of $I-\bar{T} T$ is infinite dimensional which is a contradiction. Hence $T \in P(X)$. This completes the proof.

COROLLARY 2.1. If $X$ is any subprojective space, then $S(X)=I(X)=P(X)$.

Proof. Since $X$ is subprojective, by theorem 1.3 of $[4], P(X)=S(X)$. Hence the result follows.

COROLlary 2.2 If $X$ is reflexive and superprojective then $S C(X)=I(X)=$ $P(X)$.

Proof. For any Banach space we have

$$
S C(X) \subseteq F(X)=I(X) \quad(\text { sæe }[10]) .
$$

Hence $S C(X) \subseteq I(X) \subseteq|I(X)| P(X)$ by theorem 2.1 .

Now let $T \in P(X)$. Then $T^{*} \in P\left(X^{*}\right)$ by corollary 1.4 of [4]. Now since $X^{*}$ is subprojective ([11], p. 257), $T^{*} \in S\left(X^{*}\right)$ by corollary 2.1 and hence $T \in S C(X)$ $\{[3]$, prop. 3(a)\}. Thus $S C(X)=I(X)=P(X)$.

Throughout this paper, by $L_{p}$ we shall mean the spaces $L_{p}(S, \Sigma, \mu)$ where $S=$ $[0,1], \Sigma$ is the family of Lebesgue measurable subsets of $S$ and $\mu$ is Lebesgue measure.

Corollary 2.3. If $X=L_{n}(1<p<\infty)$, then $P(X)=I(X)$ and more over, $S C(X)=I(X)$ for $1<p \leqq 2$ and $S(X)=I(X)$ for $2 \leqq p<\infty$.

PRoOF. To prove this corollary we only need to note that $L_{p}$ is reflexive and 
superprojective for $1<P \leqq 2$ and subprojective for $2 \leqq p<\infty$ and then apply corollary 2.2 and 2.1 respectively.

Remark. We should point out that the corollary 2.1 has been proved by Pfaffenberger ([7],Th. 2.2) and the part of the corollary 2.3 that $S(X)=F(X)$ for $p \geqq 2$ has been announced by Milman [12].

Riesz Operators. An operator $T \in B(X)$ is called a Riesz operator if $T-\lambda I \in \phi(X)$ for all scalars $\lambda \neq 0$. Let $R(X)$ denote the class of all Riesz operators on $X$. We can easily see that $R(X)$ is not always included in $P(X)$. For example, if $X$ is subprojective and $R(X) \subseteq P(X)$ then $R(X) \subseteq P(X)=S(X)$. Also $S(X) \in R(X)$ for every Banach space $X$ [13] p. 66). Hence $S(X)=R(X)$. This implies that $R(X)$ is a closed ideal in $B(X)$ when $X$ is a subprojective space. But this is impossible when $X=l$ which is a subprojective space (see [13], remark p. 67-68).

Whether or not $P(X) \subset R(X)$ for every Banach space remains open. We have, however, the following proposition:

Proposition 2.1. If for some Banach space $X, P(X) \subseteq R(X)$ then $P(X)=$ $I(X)$.

Pkcof. Let $T \in P(X)$. Then for every $T_{1} \in B(X)$, both $T T_{1}$ and $T_{1} T$ are in $P(X)$ (see [4], th.1.2). Now since every member of $R(X)$ has Riesz spectrum (for definition and proof see [13], p.66), both $T T_{1}$ and $T_{1} T$ have Riesz spectrum by the condition of the proposition. Now proceeding exactly as in the proof of Theorem 1 of [8] we can show that $T \in I(X)$ (note that the linearity of the ideal in theorem 1 of [8] is not used). Thus $P(X) \subset I(X)$. The rest of the proof follows our theorem 2.1.

We have already defined the notion of an ideal in a category of Banach spaces in [4]. If we now consider the category of a single infinite dimensional Banach space $X$ with all the bounded liner operators on $X$ as morphisms, then by theorem 1.2 of [4] $P(X)$ is an ideal in this category. In other words, $P(X)$ is a two-sided ideal in the semigroup $B(X)$ of all bounded linear operators on $X$; in this case we are not considering $B(X)$ as a ring as we do not yet know if the sum of two improjective operators is improjective. However, it is trivial that every two-sided ideal in the ring $B(X)$ is also a two-sided ideal in the semigroup $B(X)$.

THEOREM 2.2. If $X$ is a Banach space such that every infinite dimensional complemented subspace of $X$ contains a subspace which is complemented in $X$ and isomorphic to $X$, then $P(X)$ is the largest ideal in the semigroup $B(X)$.

Proof. Since $X$ is infinite dimensional, $P(X)$ is a proper ideal in $B(X)$. We will assert that any ideal $I$ which is not contained in $P(X)$ must coincide with $B(X)$. Let $T$ be any member of $I$ such that $T \notin P(X)$. Then there is an infinite dimensional closed subspace $M$ of $X$ such that the restriction of $T$ to $M$ is 
an isomorphism and $T M$ is complemented in $X$. Now by the hypothesis of the theorem $T M$ contains an infinite dimensional subspace $M^{\prime}$ such that $M^{\prime}$ is isomorphic to $X$ and complemented in $X$. Let $J$ be an isomorphism of $M^{\prime}$ onto $X$ and $Q$ be a projection of $X$ onto $M^{\prime}$. We set $T^{-1}\left(M^{\prime}\right) \cap M=N$. Then the restriction of $T$ to $N$ is also an isomorphism, i.e., $N$ and $M^{\prime}$ are isomorphic. Hence $N$ is isomorphic to $X$. Let $U$ be an isomorphism of $X$ onto $N$. Then $i . U \in B(X)$ where $i$ is the injection map of $N$ into $X$. Also $J . Q \in B(X)$. We have the following diagram:

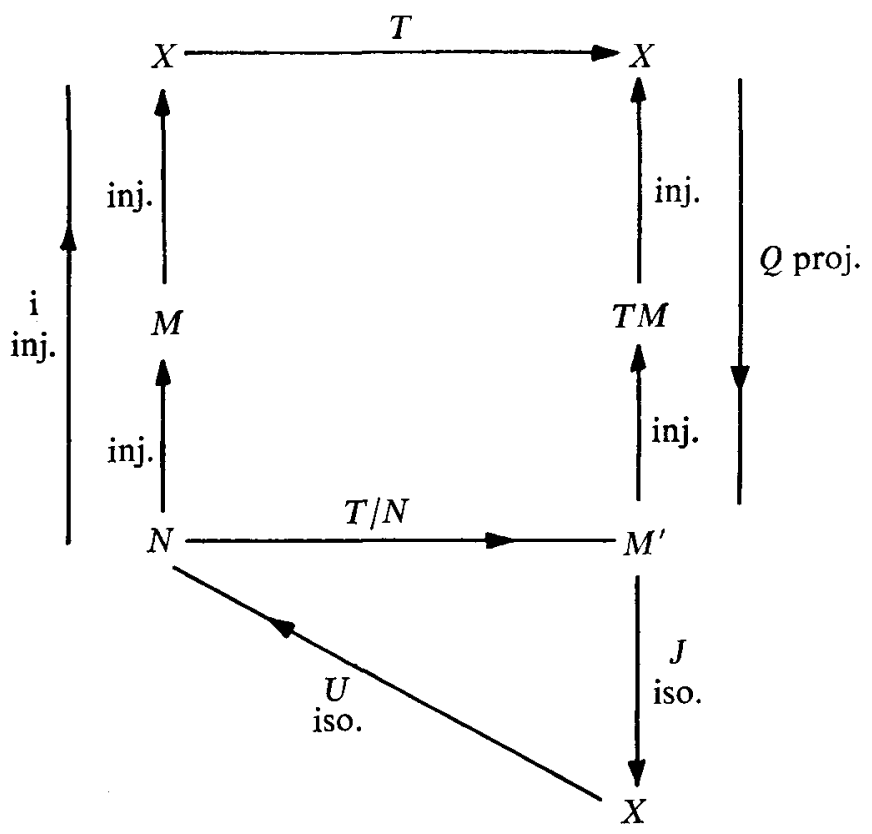

We consider the composition map $T_{1}=J Q T i U$ which is $=J T / N U$ and clearly an isomorphism of $X$ onto $X$. Now $T_{1} \in I$ as $T \in I, J Q \in B(X)$ and $i U \in B(X)$. Hence $I_{X}=T_{1}^{-1} T_{1} \in I$ as $T_{1} \in I$ and $T_{1}^{-1} \in B(X), I_{X}$ being the identity operator on $X$. Thus $I=B(X)$. This proves the theorem.

Now since the spaces $l^{p}(1 \leqq P<\infty), c_{0}$ and $m$ satisfy the hypothesis of the above theorem (see [14] and [15]) we have the following corollary.

Corollary 2.4. If $X=l P\left(I \leqq P^{p}<\infty\right), c_{0}, o r, m$, then $P(X)$ is the largest ideal in the semigroup $B(X)$.

REMARK. For the spaces $l^{p}(I \leqq p<\infty)$ and $c_{0}$ stronger result is already known [5].

3.

Definition. A Banach space $X$ will be said to be totally dissimilar with a Banach space $Y$ if no infinite dimensional complemented subspace of $X$ is isomor- 
phic to a complemented subspace of $Y$. In this case we will say that $X$ and $Y$ are totally dissimilar.

THEOREM 3.1. The following paris are totally dissimilar:

(i) $X, Y$ where $X \in P$ and $Y$ is a Banach space isomorphic to an abstract L-space.

(A Banach space $X$ is in $P$, or $X \in P$ if $X$ is complemented in every Banach space which contains it.)

(ii) $C(S), X$ where $S$ is a compact Hausdorff topological space and $X$ is a Banach space isomorphic to an abstract L-space.

(iii) $X, Y$ where $X$ is a Banach space isomorphic to an abstract L-space and and $Y=c_{0}$, or a reflexive Banach space.

(iv) $X, Y$ where $Y$ is a reflexive Banach space and $X$ is either of $(a) C(S)$ where $S$ is a compact Hausdorff space, (b) $B(S), S$ a set, (c) $L_{\infty}(S, \Sigma, \mu)$ or $L(S, \Sigma, \mu)$ where $(S, \Sigma, \mu)$ is a positive measure space, $(d) r c a(S)$ or ba(S) and (e) any infinite dimensional complemented subspace of $(a)$ to $(d)$.

(v) $X, Y$ where $X \in P$ and $Y$ is either a separable or a reflexive Banach space.

(vi) $l^{p}(1 \leqq p<\infty), c_{0}$. and (vii) $l^{p}, l^{q}(1 \leqq p, q<\infty$ and $p \neq q)$.

Proof. (i) If possible, let an infinite dimensional complemented subspace $Z$ of $X$ be isomorphic to a complemented $U$ of $Y$. Then since $Y$ is isomorphic to an abstract $\mathrm{L}$-space, $U$ contains a complemented subspace $V$ isomophic to $l([14]$, cor. 4) ( $V$ is complemented in $Y$ as well as in $U$ ). Let $T$ be an isomorphism of $U$ onto $Z$. Then $T V$ is complemented in $Z$. Hence $T V$ is complemented in $X$ as $Z$ is complemented in $X$. Now since $X \in P$, by a result of Goodner ([16], p.93) $T V \in P$ (this can also be proved by using the result that a Banach space $X \in P$ is and only if $X$ is isomorphic to a complemented subspace of a space $B(S)$; see [11], p. 256). $T V$ being isomorphic to $V$ is isomorphic to $l$. Hence $T V$ is separable and $T V \in P$. Then by a result of Grothendieck ([17], p. 169) (also [14], Th. 6), $T V$ is finite dimensional which is a contradiction.

(ii) The proof of theorem 2.4 in [4] contains a proof of (ii).

(iii) Suppose that an infinite dimensional complemented subspace $Z$ of $X$ is isomorphic to a complemented subspace $U$ of $Y$. Then as in (i) $Z$ contains a complemented subspace $R$ isomorphic to $l$. If $Y$ is $c_{0}$, then $U$ is isomorphic to $c_{0}$ ([14], Th. 1). Now since $Z$ is isomorphic to $U$, the above will imply that $c_{0}$ contains a subspace isomorphic to $l$ which is impossible.

If $Y$ is reflexive, then $U$ is also reflexive. But this would imply that $U$ contains a subspace isomorphic to $l$ which is again impossible as $U$ is reflexive.

(iv) This follows from lemma 2.1 of [4].

(v) If possible, let an infinite dimensional complemented subspace $Z$ of $X$ be isomorphic to a closed subspace $U$ of $Y$ (in this case we do not need $U$ to be 
complemented in $Y$ ). Since $X \in P$, and $Z$ is complemented in $X, Z \in P$ by the reason given in (i). Now $U$ is separable or reflexive according as $Y$ is separable or reflexive. Hence $Z$, being isomorphic to $U$, is either separable or retlexive. Then in either case $Z$ is finite dimensional by the result of Grothendieck quoted in (i), as $Z \in P$. This is a contradiction. (vi) and (vii) are simple consequences of theorem 1 [14].

THEOREM 3.2 Let $X$ and $Y$ be any two Banach spaces. Then $B(X Y)=P$ $(X, Y)$ if and only if $X$ and $Y$ are totally dissimilar.

Proof. First, let $X$ and $Y$ be totally dissimilar. Let $T: X \rightarrow Y$ be an arbitrary bounded liner operator. Suppose that $T \notin P(X, Y)$. Then there is an infinite dimensional closed subspace $M$ of $X$ such that the restriction of $T$ to $M$ is an isomorphism and $T M$ is complemented in $Y$. Also by lemma 1.1 of [4], $M$ is complemented in $X$. Thus the infinite dimensional complemented subspace $M$ of $X$ is isomorphic to the complemented subspace $T M$ of $Y$. This contradicts that $X$ and $Y$ are totally dissimilar. Hence we conclude that $B(X, Y)=P(X, Y)$. Next, let $B(X, Y)$ $=P(X, Y)$. If possible, let $X$ and $Y$ be not totally dissimilar. Then there must be an infinite dimensional complemented subspace $Z$ of $X$ and in infinite dimensional complemented subspace $U$ of $Y$ such that $Z$ and $U$ are isomorphic. Let $j$ be an isomorphism of $Z$ onto $U$. Now since $Z$ is complemented in $X$, there is a projection $P$ of $X$ onto $Z$. Let us now define $\hat{T}: X \rightarrow Y$ by $\hat{T}=i j P$ where $i$ is the injection map of $U$ into $Y$. Clearly $\hat{T} \in B(X, Y)$. It is easy to see that the restriction of $\hat{T}$ to $Z$ is an isomorphism and $\hat{T} Z=U$ is complemented in $Y$. Hence $\hat{T} \notin P$ $(X, Y)$. But this contradicts the hypothesis that $B(X, Y)=P(X, Y)$. Hence $X$ and $Y$ are totally dissimilar.

CoRollary 3.1. If $X$ and $Y$ are toally dissimilar Banach spaces, then (i) $B(X, Y)=S(X, Y)$ if $Y$ is subprojective;

and (ii) $B(X, Y)=S C(X, Y)$ if $X$ is reflexive and superprojective.

Proof. Since $X$ and $Y$ are totally dissimilar, we have by theorem $3.2 B(X, Y)$ $=P(X, Y)$. Then by theorem 1.3 of $[4]$,

$$
B(X, Y)=S(X, Y) \text { if } Y \text { is subprojective. }
$$

This proves (i).

Now let $X$ be reflexive and superprojective and $T: X \rightarrow Y$ be any bounded linear operator of $X$ into $Y$. Then $T \in B(X, Y)=P(X, Y)$. Since $X$ is reflexive, $T^{*}: Y^{*} \rightarrow X^{*}$ is improjective by corollary 1.4 of [4]. Again since $X$ is reflexive and superprojective, $X^{*}$ is subprojective by a result of Whitely ([11], cor. 4.7, p. 257). Thus $T^{*}$ is strictly singular by theorem 1.3 of [4]. Hence by a result of Pelczynski ([3], prop. 3(a)) $T$ is strictly cosingular. Thus $B(X, Y)=S C(X, Y)$. This proves (ii). 
Remark. We point out that although a recent result (Theorem 4) of Milman [12] is similar to the part (i) of our cor. 3.1, ours involve weaker hypotheses.

COROLlaRY. 3.2. If $X$ is a Banach space isomorphicto an abstract $L$-space and $Y=c_{0}$, or $l^{p}(S)(1<p<\infty)$ or $L_{p}(2 \leqq p<\infty)$ or $C\left(S_{1}\right)$ where $S_{1}$ is metrizable compact and dispersed, then $B(X, Y)=S(X, Y)$.

Proof. $X$ and $Y$ are totally dissimilar by theorem 3.1 (ii) when $Y=C\left(S_{1}\right)$ and by theorem 3.1 (iii) when $Y$ is other than $C\left(S_{1}\right)$. Also $Y$ is subprojective (see [11] and [4]).

Hence $B(X, Y)=S(X, Y)$ by corollary 3.1 (i).

COROLlary 3.3 If $X$ is a Banach space isomorphic to an abstract L-space and

$$
Y=l^{p}(S)(1<p<\infty) \text { or } L_{p}(1<p \leqq 2),
$$

then $B(Y, X)=S C(Y, X)$.

Proof. $X$ and $Y$ are totally dissimilar as in corollary 3.2 and $Y$ is reflexive and superprojective [11]. Hence $B(Y, X)=S C(Y, X)$ by corollary 3.1 (ii).

CoRollary 3.4. If $X \in P$ and $Y=c_{0}(S), S$ being a countable set, $l^{p}\left(S_{1}\right)$ $(1<\mathrm{p}<\infty)$ or $l\left(S_{2}\right), S_{2}$ being a countable set or $L_{p}(2 \leqq P<\infty)$ or $C\left(S^{\prime}\right), S^{\prime}$ being metrizable compact and dispersed, then

$$
B(X, Y)=S(X, Y)=W(X, Y) .
$$

Proof. $X$ and $Y$ are totally dissimilar by theorem 3.1 (v) as $Y$ is either a separable or a reflexive Banach space. Also $Y$ is subprojective. Hence $B(X, Y)$ $=S(X, Y)$.

Now since every Banach space can be embedded in a space $C(S), S$ being compact and Hausdorff and $X \in P$, is isomorphic to a complemented subspace of $C(S)$. Hence $X$ is a continuous linear image of $C(S)$, i.e., there is a continuous linear operator of $C(S)$ onto $X$. Hence by proposition $4(2)$ of [18] $S(X, Y) \subset W$ $(X, Y)$, Thus the corollary is proved.

Corollary 3.5. If $X \in P$ and $Y=l^{p}(S)(1<p<\infty)$ or $L_{p}(1<p \leqq 2)$, then $B(Y, X)=S C(Y, X)$.

Proof. $X$ and $Y$ are totally dissimilar by theorem 3.1 (v) and $Y$ is reflexive and superprojective. Hence the corollary follows from corollary 3.1 (ii).

A Banach space $X$ is said to belong to $P_{1}$ or said to be in $P_{1}$, if given any Banach space $Z$ containing $X$ there is a projection $P$ of $Z$ onto $X$ with $\|P\|=1$.

CoRollaRY 3.6. If $X \in P_{1}$ and $Y=$ any reflexive Banach space, or $c_{0}(S)$, $S$ being a countable set, or $l\left(S_{1}\right), S_{1}$ being a countable set or $C\left(S^{\prime}\right), S^{\prime}$ being metrizable compact and dispersed, then $B(X, Y)=S(X, Y)=W(X, Y)$. 
Proof. If $T=c_{0}(S)$, or $l\left(S_{1}\right)$, or $C\left(S^{\prime}\right)$, then the proof is immediate from corollary 3.4 .

Suppose that $X$ is any reflexive Banach space. Since $X \in P_{1}$, it is known ([19], p. 95) that $X$ is isometrically isomorphic to a space $C(S)$ where $S$ is compact Hausdorff and extremally disconnected. Let $j$ be any isometry of $C(S)$ onto $X$. Now let $T$ be any bounded linear operator of $X$ into $Y$. Then $T j=T^{\prime}$, say, is a bounded linear operator of $C(S)$ into $Y$. But then by a result of Whitley ([11], p. 253) $T^{\prime}$ is strictly singular. Hence $T=T^{\prime} j^{-1}$ is strictly singular. Thus $B(X, Y)=S(X, Y)$. The rest is trivial (e.g. see the proof of corollary 3.4).

REMARK. $B(S) \in P([11]$, p. 256$)$ and $m, U^{*}, V^{* *} \in P_{1}([16]$, p. 106) where $U$ is an abstract $L$-space and $V$ an abstract $M$-space. However, for us the interest of the corollary 3.4 for $X=B(S)$ is only for the non reflexive part of $Y$, because the following result is already known $([11]$, p. 253):

If $X$ and $Y$ are as in theorem 2.1 (iv), then $B(X, Y)=S(X, Y)$.

Corollary 3.7. If $X=L_{\infty}(S, \Sigma, \mu)$ where $(S, \Sigma, \mu)$ is a positive measure space, or $\operatorname{rca}(S)$ or $b a(S)$ and

$$
Y=l^{p}(S)(1<p<\infty) \text { or } L_{p}(1<p \leqq 2),
$$

then $B(Y, X)=S C(Y, X)$.

Proof. $X$ and $Y$ are totally dissimilar by theorem 3.1 (iv) and $Y$ is reflexive and superprojective. The proof now flows from corollary 3.1 (ii).

Corollary 3.8. If $X=C(S), S$ a compact Haudorff space, or $B(S), S$ a set or $L(S, \Sigma, \mu)$ where $(S, \Sigma, \mu)$ a positive measure space and $Y$ is any reflexive Banach space, then $B(Y, X)=S C(Y, X)$.

Proof. Let $T$ be any bounded linear operator $\in B(Y, X)$. Then $T^{*} \in B$ $\left(X^{*}, Y^{*}\right)$ where $X^{*}=\operatorname{rca}(S)$ or $b a(S)$ or $L_{\infty}(S, \Sigma, \mu)$ and $Y^{*}$ is a reflexive Banach space. Hence by the result of [11] quoted in the above remark, $T^{*} \in S\left(X^{*}, Y^{*}\right)$.

Therefore $T \in S C(Y, X)$. This proves the corollary.

Lemma 3.1 The pair $L_{p}, l^{q}(1 \leqq p<\infty, 1 \leqq q<\infty, q \neq 2$ and $p \neq q)$ is totally dissimlar.

Proof. Since $L$ is an abstract $L$-space, the pair $L, l^{q}(1<q<\infty)$ is totally dissimilar by theorem 2.1 (iii). Let us now consider the pair $L_{p}, l^{q}$ where $p$ and $q$ satisfy $1<p<<\infty, 1 \leqq q<\infty, q \neq 2$ and $p \neq q$.

If possible, let an infinite dimensional complemented subspace $M$ of $L_{p}$ be isomorphic to a complemented subspace $N$ of $l^{q}$. Then by theorem 1 of [14], $N$ is isomorphic to $l^{q}$. Now since $M$ is complemented in $L_{p}(1<p<\infty)$, either $M$ 
is isomorphic to $l^{2}$, or $M$ contains a subspace isomorphic to $l^{p}([20]$, p. 168$)$. But the fact that $M$ is isomorphic to $l^{2}$ will imply that $l^{2}$ is isomorphic to $l^{p}$ where $q \neq 2$. This is impossible ([21], p. 205). Again the fact that $M$ contains a subspace isomorphic to $l^{p}$ will imply that $l^{q}$ contains a subspace isomorphic to $l^{p}$ where $p \neq q$. This is also impossible ([21], p. 205). Thus we have proved the lemma.

Because $l^{q}(1 \leqq q<\infty)$ is subprojective and $l^{q}(1<q<\infty)$ is reflexive and superprojective, the following corollary is an immediate consequence of the above lemma 3.1 and the corollary 3.1 .

Corollary 3.9. $B\left(L_{p}, l^{q}\right)=S\left(L_{p}, l^{q}\right)$ where $1 \leqq p<\infty, 1 \leqq q<\infty, q \neq 2$ and $p \neq q$ and $B\left(l^{q}, L_{p}\right)=S C\left(l^{q}, L_{p}\right)$ where $1 \leqq p<\infty, 1<q<\infty, q \neq 2$ and $p \neq q$.

THeOREM 3.3. An operator $T \in B\left(L_{p}, L_{q}\right)(1<p, q<\infty, p \neq q)$ is improjective if the restriction of $T$ to a subspace $M$ (of $L_{p}$ ) isomorphic to $l^{2}$ is never an isomorphism of Monto TM.

Proof. Suppose that the condition of the theorem holds. If possible, let $T$ be not improjective. Then there is an infinite dimensional closed subspace $M$ of $L_{p}$ such that the restriction of $T$ to $M$ is an isomorphism onto $T M$ and $T M$ is complemented in $L_{q}$. Since $T M$ is an infinite dimensional complemented subspace of $L_{q}$, then either $T M$ is isomorphic to $l^{2}$ or $T M$ contains a subspace isomorphic to $l^{q}$ and complemented in $L_{q}$ ([20], p. 168). Now if $T M$ is isomorphic to $l^{2}$, then $M$ being isomorphic to $T M$ will be isomorphic to $l^{2}$ which will contradict the hypothesis of the theorem. Hence we are left with the alternative that $T M$ contains a subspace $M_{1}$ isomorphic to $l^{q}$ and complemented in $L_{q}$. We set $T^{-1}\left(M_{\mathrm{i}}\right) \cap M$ $=N$. Then since the restriction of $T$ to $N$ is also an isomorphism and $T N=M_{1}$ is complemented in $L_{q}$, by lemma 1.1 of [4], $\mathrm{N}$ is complemented in $L_{p}$. But then by the same result of [20] quoted above, either $N$ is isomorphic to $l^{2}$ or $N$ contains a subspace isomorphic to $l^{p}$. We now prove that neither of these two alternatives is true. Clearly $\mathrm{N}$ can not be isomorphic to $l^{2}$ by the condition of the theorem. Nor could $N$ contain a subspace isomorphic to $l^{p}$, because if $N$ contains a subspace isomorphic to $l^{p}$, then the fact that $N$ is isomorphic to $M_{1}$ would imply that $l^{q}$ contains a subspace isomorphic to $l p$ where $q \neq p$ which is impossible by the reason given earlier.

Corollary 3.10. An operator $T \in B\left(L_{p}, L_{q}\right)(1<p<\infty, 2 \leqq q<\infty$, $p \neq q$ ) is strictly singular if and only if the restriction of T to a subspace (of $L_{p}$ ) isomorphic to $l^{2}$ is never an isomorphism.

Proof. Since $L_{q}, 2 \leqq q<\infty$ is subprojective, the corollary follows from the above theorem. 


\section{References}

[1] T. Kato, 'Perturbation theory, nullity, deficiency and other quantities of linear operators', J. Analyse Math. 6 (1958), 261-322.

[2] S. Goldberg, Unbounded linear operators, Theory and applications, (McGraw-Hill (1966).

[3] A. Pelczynski, 'On strictly singular and strictly cosingular operators I and II', Bull. Acad. Polon. Sci. 13 (1965), 31-41.

[4] E. Tarafdar, 'Improjective operators and ideals in a category of Banach spaces', J. Austral. Math. Soc. 14 (1972), 274-292.

[5] I. C. Cohberg, A. S. Markus and I. A. Feldman, 'Normally soluble operators and ideals associated with them Iav. Moldavask.' (Russian). Fil. Akad. Nauk SSSR 10, no. 76 (1960), 51-59.

[6] N. Dunford and J. Schwartz, Linear operators I: General theory, Pure and Applied Math. vol. 7, (Interscience, New York, (1958).)

[7] W. Pfaffenberger, 'On the ideals of strictly singular and inessential operators,' Proc. Amer. Math. Soc. 25 (1970), 603-607.

[8] D. Kleinecke, 'Almost-finite, compact, and inessential operators', Proc. Amer. Math. Soc. 14 (1963), 863-868.

[9] M. Schechter, 'Riesz operators and Fredholm perturbations', Bull. Amer. Math. Soc. 74 (1968) 1139-1144.

[10] Ju. N. Vladimirskii, 'Strictly consingular operators', Soviet Math. Dokl. vol. 8 no. 3 (1967), 739-740.

[11] R. J. Whitley, 'Strictly singular operators and their conjugates', Trans. Amer. Math. Soc. 113 (1964), 252-261.

[12] V. D. Milman, 'Certain properties of strictly singular operators' (Russian) Funkeional Anal. i pritozen 3, no. 1 (1969), 93-94 MR 3973332.

[13] S. R. Caradus, 'Operators of Riesz type', Pacific J. Math. 18 (1966), 61-71.

[14] A. Pelczynski, 'Projections in certain Banach spaces', Studia Math. 19 (1960), 209-228.

[15] J. Lindesntrauss, 'On complemented subspaces of $m$ ', Israel J. Math. 5 (1967), 153-156.

[16] D. B. Goodner, 'Projections in normed linear spaces', Trans. Amer. Math. Soc. 69 (1950), 89-108.

[17] A. Grothendieek, 'Sur les applications linéaires faiblement compacts d'espaces du type $C(K)$ ', Canad. J. Math. 5 (1953), 129-173.

[18] H. E. Lacey and P. D. Morris, 'Continuous linear operators on spaces of continuous functions,' Proc. Amer. Math. Soc. 17 (1966), 848-853.

[19] M. Day, Normed linear spaces (Springer, Berlin (1958)).

[20] M. Kadec and A. Pelczynski, 'Bases, lacunary sequences and complemented subspaces in the spaces $L_{p}$,' Studia Math. 21 (1962), 161-176.

[21] S. Banach, Opérations linéaires (Chelsea, New York (1955)).

School of Mathematical Sciences

Flinders University

South Australia

Present address.

Department of Mathematics

University of Queensland

St. Lucia Brisbane

Australia 